\title{
Modulus Fault Attacks against RSA-CRT Signatures
}

\author{
Éric Brier ${ }^{1}$, David Naccache ${ }^{2}$, Phong Q. Nguyen ${ }^{2}$, and Mehdi Tibouchi ${ }^{2}$ \\ 1 Ingenico \\ 1, rue Claude Chappe, BP 346, F-07503 Guilherand-Granges, France \\ eric.brier@ingenico.com \\ 2 École normale supérieure \\ Département d'informatique, Groupe de Cryptographie \\ 45, rue d'Ulm, F-75230 Paris Cedex 05, France \\ \{david.naccache, phong.nguyen, mehdi.tibouchi\}@ens.fr
}

\begin{abstract}
RSA-CRT fault attacks have been an active research area since their discovery by Boneh, DeMillo and Lipton in 1997. We present alternative key-recovery attacks on RSA-CRT signatures: instead of targeting one of the sub-exponentiations in RSA-CRT, we inject faults into the public modulus before CRT interpolation, which makes a number of countermeasures against Boneh et al.'s attack ineffective.

Our attacks are based on orthogonal lattice techniques and are very efficient in practice: depending on the fault model, between 5 to 45 faults suffice to recover the RSA factorization within a few seconds. Our simplest attack requires that the adversary knows the faulty moduli, but more sophisticated variants work even if the moduli are unknown, under reasonable fault models. All our attacks have been fully validated experimentally with fault-injection laser techniques.
\end{abstract}

Keywords: Fault Attacks, Digital Signatures, RSA, CRT, Lattices.

\section{Introduction}

\subsection{RSA-CRT Signatures}

RSA 23 is the most widely used signature scheme. To sign a message $m$, the signer first applies an encoding function $\mu$ to $m$, and then computes the signature $\sigma=\mu(m)^{d} \bmod N$. To verify the signature $\sigma$, the receiver checks that $\sigma^{e}=\mu(m)$ $\bmod N$. The Chinese Remainder Theorem $(\mathrm{CRT})$ is often used to speed up signature generation by a factor of about 4 . This is done by computing:

$$
\sigma_{p}=\mu(m)^{d \bmod p-1} \bmod p \quad \text { and } \quad \sigma_{q}=\mu(m)^{d \bmod q-1} \bmod q
$$

and deriving $\sigma$ from $\left(\sigma_{p}, \sigma_{q}\right)$ using the CRT. 


\subsection{Fault Attacks on RSA-CRT Signatures}

Back in 1997, Boneh, DeMillo and Lipton [6] showed that RSA-CRT implementations are vulnerable to fault attacks. Assuming that the attacker can induce a fault when $\sigma_{q}$ is computed while keeping the computation of $\sigma_{p}$ correct, one gets:

$$
\sigma_{p}=\mu(m)^{d \bmod p-1} \quad \bmod p \quad \text { and } \quad \sigma_{q} \neq \mu(m)^{d \bmod q-1} \quad \bmod q
$$

hence:

$$
\sigma^{e}=\mu(m) \quad \bmod p \quad \text { and } \quad \sigma^{e} \neq \mu(m) \quad \bmod q
$$

which allows the attacker to factor $N$ by computing $\operatorname{gcd}\left(\sigma^{e}-\mu(m) \bmod N, N\right)=$ $p$. This attack applies to any deterministic padding function $\mu$, such as RSA PKCS\#1 v1.5 or Full-Domain Hash [2, or probabilistic signatures where the randomizer used to generate the signature is sent along with the signature, such as PFDH 13. Only probabilistic signature schemes such that the randomness remains unknown to the attacker may be safe, though some particular cases have been attacked as well [12.

In 2005, Seifert 24] introduced a new type of RSA fault attacks, by inducing faults on the RSA public modulus. The initial attack 24] only allowed to bypass RSA verification, but key-recovery attacks were later discovered by Brier et al. [8, and improved or extended in [17/5/3/4. These key-recovery attacks only apply to RSA without CRT, and they require significantly more faults than Boneh et al.'s attack, at least on the order of 1000 faulty signatures.

\subsection{Our Contribution}

We present new alternative key-recovery attacks on RSA-CRT signatures: instead of targeting one of the RSA-CRT sub-exponentiations, we inject faults into the public modulus like in Seifert's attack. This makes typical countermeasures against Boneh et al.'s attack ineffective against the new attacks.

Our attacks are based on the orthogonal lattice techniques introduced by Nguyen and Stern [19] in 1997. They are very effective in practice: they disclose the RSA factorization within a few seconds using only between 5 to 45 faulty signatures. The exact running time and number of faulty signatures depend on the fault model.

For instance, in our simplest attack, the running time is a fraction of a second using only 5 faulty signatures, but the attacker is assumed to know the faulted moduli for the 5 different messages. However, our attack can be extended to the case where the attacker no longer knows the faulted moduli, using at most 45 faulty signatures, under the following two fault models: either the faulted moduli only differ from the public modulus on a single byte of unknown position and unknown value, or the faulted moduli may differ from the public modulus by many bytes, but the differences are restricted to the least significant bits, up to half of the modulus size.

All our attacks have been fully validated with physical experiments with laser shots on a RISC microcontroller. 


\subsection{Related Work}

Many countermeasures have been proposed to protect against Boneh et al.'s attack and its numerous generalizations, but they often focus on the exponentiation process. The previously mentioned fault attacks 817534 on RSA using faulty moduli only apply to standard RSA without CRT, and they use nonlattice techniques. Our attack seems to be the first attack on RSA-CRT with faulted moduli.

It should be pointed out, however, that a number of protected RSA-CRT implementations also protect the CRT recombination. This is for example the case of $110 / 147 / 2622$.

More generally, as we observe in \$5, using the technique known as Garner's formula for CRT recombination does thwart the attack introduced in this paper. Since this formula is often used in practice, typical implementations conforming to RSA standards like PKCS\#1 and IEEE P1363 should in principle be immune to this attack.

\subsection{Roadmap}

In $\$ 2$, we describe the basic attack where the faulty moduli are assumed to be known to the attacker. In 93 , we extend the attack to realistic fault models in which the faulty moduli are no longer known to the attacker. In $\$ 4$, we describe physical experiments with laser shots on a RISC microcontroller to validate the attack. Finally, in \$5, we suggest possible countermeasures against this attack.

\section{The New Attack}

\subsection{Overview}

Consider again the generation of RSA-CRT signatures. To obtain the signature $\sigma$ of a message $m$ padded as $\mu(m)$, the signer computes the mod- $p$ and mod- $q$ parts:

$$
\sigma_{p}=\mu(m)^{d} \bmod p \quad \text { and } \quad \sigma_{q}=\mu(m)^{d} \bmod q
$$

and returns the signature:

$$
\sigma=\sigma_{p} \cdot \alpha+\sigma_{q} \cdot \beta \bmod N
$$

where $\alpha, \beta$ are the pre-computed Chinese Remainder coefficients $\alpha=q \cdot\left(q^{-1} \bmod \right.$ $p)$ and $\beta=p \cdot\left(p^{-1} \bmod q\right)$.

Assume that an adversary can obtain the correct signature $\sigma$, and also a signature $\sigma^{\prime}$ of the same padded message $\mu(m)$ after corrupting the modulus $N$ before the CRT step (1). In other words, the attacker gets $\sigma$ as before but also $\sigma^{\prime}$ defined as:

$$
\sigma^{\prime}=\sigma_{p} \cdot \alpha+\sigma_{q} \cdot \beta \quad \bmod N^{\prime} \quad \text { for some } N^{\prime} \neq N
$$


Suppose further, for the moment, that the adversary is able to recover the faulty modulus $N^{\prime}$ : we will see in $\$ 3$ how this not-so-realistic hypothesis can be lifted in a more practical setting. Then, by applying the Chinese Remainder Theorem to $\sigma$ and $\sigma^{\prime}$, the adversary can compute

$$
v=\sigma_{p} \cdot \alpha+\sigma_{q} \cdot \beta \bmod N \cdot N^{\prime}
$$

But if we denote the bit length of $N$ by $n$, then $N \cdot N^{\prime}$ is a $2 n$-bit integer, whereas $\alpha, \beta$ are of length $n$ and $\sigma_{p}, \sigma_{q}$ of length $n / 2$, so $v$ is really a linear combination of $\alpha$ and $\beta$ in $\mathbb{Z}$ :

$$
v=\sigma_{p} \cdot \alpha+\sigma_{q} \cdot \beta
$$

That alone does not suffice to factor $N$, but several such pairs $\left(\sigma, \sigma^{\prime}\right)$ provide multiple linear combinations of the (unknown) integers $\alpha, \beta$ with relatively small coefficients. Then lattice reduction techniques allow us to recover the coefficients $\sigma_{p}$ and $\sigma_{q}$, and hence obtain the factorization of $N$ by GCDs. The following sections describe this process in detail.

\subsection{Applying Orthogonal Lattice Techniques}

We assume that the reader is familiar with cryptanalysis based on lattices (see [1821] for more information), particularly the orthogonal lattices introduced by Nguyen and Stern [19]: if $L$ is a lattice in $\mathbb{Z}^{n}$, we let $L^{\perp}$ be the lattice formed by all vectors in $\mathbb{Z}^{n}$ which are orthogonal to all vectors of $L$. If an attacker obtains $\ell$ pairs $\left(\sigma, \sigma^{\prime}\right)$, he can compute as before a vector $\boldsymbol{v}=\left(v_{1}, \ldots, v_{\ell}\right)$ of $3 n / 2$-bit integers satisfying an equation of the form:

$$
\boldsymbol{v}=\alpha \boldsymbol{x}+\beta \boldsymbol{y}
$$

where $\boldsymbol{x}, \boldsymbol{y}$ are unknown vectors with $n / 2$-bit components and $\alpha, \beta$ are the (unknown) CRT coefficients relative to $p$ and $q$. Lattice reduction can exploit such a hidden linear relationship as follows.

Using standard techniques 1920, it is possible to compute a reduced basis $\left\{\boldsymbol{b}_{1}, \ldots, \boldsymbol{b}_{\ell-1}\right\}$ of the lattice $\boldsymbol{v}^{\perp} \subset \mathbb{Z}^{\ell}$ of vectors orthogonal to $\boldsymbol{v}$ in $\mathbb{Z}^{\ell}$. In particular we get:

$$
\alpha\left\langle\boldsymbol{b}_{j}, \boldsymbol{x}\right\rangle+\beta\left\langle\boldsymbol{b}_{j}, \boldsymbol{y}\right\rangle=0 \text { for } j=1,2, \ldots, \ell-1 .
$$

Now, observe that the smallest nonzero solution $(u, v) \in \mathbb{Z}^{2}$ of the equation $\alpha \cdot u+\beta \cdot v=0$ is $\pm(\beta,-\alpha) / g$, where $g=\operatorname{gcd}(\alpha, \beta)$ is heuristically expected to be very small, which implies that $|u|,|v| \geq \Omega(N)$ where the $\Omega()$ constant is very small. For each $j=1,2, \ldots, \ell-1$, there are thus two possibilities:

Case 1: $\left\langle\boldsymbol{b}_{j}, \boldsymbol{x}\right\rangle=\left\langle\boldsymbol{b}_{j}, \boldsymbol{y}\right\rangle=0$, in which case $\boldsymbol{b}_{j}$ belongs to the lattice $L=$ $\{\boldsymbol{x}, \boldsymbol{y}\}^{\perp}$ of vectors in $\mathbb{Z}^{\ell}$ orthogonal to both $\boldsymbol{x}$ and $\boldsymbol{y}$;

Case 2: $\left\langle\boldsymbol{b}_{j}, \boldsymbol{x}\right\rangle$ and $\left\langle\boldsymbol{b}_{j}, \boldsymbol{y}\right\rangle$ have absolute value $\geq \Omega(N)$ with a very small $\Omega()$ constant. Since $\boldsymbol{x}, \boldsymbol{y}$ both have norm at most $\sqrt{\ell N}$, this implies $\left\|\boldsymbol{b}_{j}\right\| \geq$ $\Omega(\sqrt{N / \ell})$ by Cauchy-Schwarz. 
Since the lattice $L=\{\boldsymbol{x}, \boldsymbol{y}\}^{\perp}$ is of rank $\ell-2$, Case 1 cannot hold for all $\ell-1$ linearly independent vectors $\boldsymbol{b}_{j}$, so that the longest one $\boldsymbol{b}_{\ell-1}$ should be in Case 2 , and hence $\left\|\boldsymbol{b}_{\ell-1}\right\| \geq \Omega(\sqrt{N / \ell})$. On the other hand, the other vectors form a lattice of rank $\ell-2$ and volume:

$$
V=\operatorname{vol}\left(\mathbb{Z} \boldsymbol{b}_{1} \oplus \cdots \oplus \mathbb{Z} \boldsymbol{b}_{\ell-2}\right) \approx \frac{\operatorname{vol}\left(\boldsymbol{v}^{\perp}\right)}{\left\|\boldsymbol{b}_{\ell-1}\right\|}=\frac{\|\boldsymbol{v}\|}{\left\|\boldsymbol{b}_{\ell-1}\right\|} \leq \frac{\sqrt{\ell} \cdot N^{3 / 2}}{\Omega(\sqrt{N / \ell})}=O(\ell N)
$$

which can heuristically be expected to behave like a random lattice. In particular, we should have:

$$
\left\|\boldsymbol{b}_{j}\right\|=O\left(\sqrt{\ell-2} \cdot V^{1 /(\ell-2)}\right)=O\left(\ell^{1 / 2+1 /(\ell-2)} \cdot N^{1 /(\ell-2)}\right) \quad \text { for } j=1,2, \ldots, \ell-2 .
$$

This length is much smaller than $\sqrt{N / \ell}$ as soon as $\ell \geq 5$. Assuming that this is case, $\boldsymbol{b}_{j}$ should thus be in Case 1 for $j=1,2, \ldots, \ell-2$. This means that those vectors generate a sublattice $L^{\prime}=\mathbb{Z} \boldsymbol{b}_{1} \oplus \cdots \oplus \mathbb{Z} \boldsymbol{b}_{\ell-2}$ of full rank in $L=\{\boldsymbol{x}, \boldsymbol{y}\}^{\perp}$.

Taking orthogonal lattices, we get $\left(L^{\prime}\right)^{\perp} \supset L^{\perp}=\mathbb{Z} \boldsymbol{x} \oplus \mathbb{Z} \boldsymbol{y}$. Therefore, $\boldsymbol{x}$ and $\boldsymbol{y}$ belong to the orthogonal lattice $\left(L^{\prime}\right)^{\perp}$ of $L^{\prime}$. Let $\left\{\boldsymbol{x}^{\prime}, \boldsymbol{y}^{\prime}\right\}$ be a reduced basis of that lattice. We can enumerate all the lattice vectors in $\left(L^{\prime}\right)^{\perp}$ of length at most $\sqrt{\ell N}$ as linear combinations of $\boldsymbol{x}^{\prime}$ and $\boldsymbol{y}^{\prime}$. The Gaussian heuristic suggests that there should be roughly:

$$
\frac{\pi(\sqrt{\ell N})^{2}}{\operatorname{vol}\left(\left(L^{\prime}\right)^{\perp}\right)}=\frac{\pi \ell N}{V}=O(1)
$$

such vectors, so this is certainly feasible. For all those vectors $\boldsymbol{z}$, we can compute $\operatorname{gcd}(\boldsymbol{v}-\boldsymbol{z}, N)$. We will thus quickly find $\operatorname{gcd}(\boldsymbol{v}-\boldsymbol{x}, N)$ among them, since $\boldsymbol{x}$ is a vector of length $\leq \sqrt{\ell N}$ in $\left(L^{\prime}\right)^{\perp}$. But by definition of $\boldsymbol{v}$ we have:

$$
\boldsymbol{v}=\boldsymbol{x} \bmod p \quad \text { and } \quad \boldsymbol{v}=\boldsymbol{y} \bmod q
$$

so $\operatorname{gcd}(\boldsymbol{v}-\boldsymbol{x}, N)=p$, which reveals the factorization of $N$.

\subsection{Attack Summary}

Assume that, for $\ell \geq 5$ padded messages $\mu\left(m_{i}\right)$, we know a correct signature $\sigma_{i}$ and a signature $\sigma_{i}^{\prime}$ computed with a faulty modulus $N_{i}^{\prime}$. Then, we can heuristically recover the factorization of $N$ as follows.

1. For each $i$, compute the integer $v_{i}=\operatorname{CRT}_{N, N_{i}^{\prime}}\left(\sigma_{i}, \sigma_{i}^{\prime}\right)$. They form a vector $\boldsymbol{v}=\left(v_{1}, \ldots, v_{\ell}\right) \in \mathbb{Z}^{\ell}$.

2. Compute an LLL-reduced [15] basis $\left\{\boldsymbol{b}_{1}, \ldots, \boldsymbol{b}_{\ell-1}\right\}$ of the lattice $\boldsymbol{v}^{\perp} \subset \mathbb{Z}^{\ell}$ of vectors in $\mathbb{Z}^{\ell}$ orthogonal to $\boldsymbol{v}$. This is done by applying LLL to the lattice in $\mathbb{Z}^{1+\ell}$ generated by the rows of the following matrix:

$$
\left(\begin{array}{cccc}
\kappa v_{1} & 1 & & 0 \\
\vdots & \ddots & \\
\kappa v_{\ell} & 0 & & 1
\end{array}\right)
$$

where $\kappa$ is a suitably large constant, and removing the first component of each resulting vector [19]. 
3. The first $\ell-2$ vectors $\boldsymbol{b}_{1}, \ldots, \boldsymbol{b}_{\ell-2}$ generate a lattice $L^{\prime} \subset \mathbb{Z}^{\ell}$ of rank $\ell-2$. Compute an LLL-reduced basis $\left\{\boldsymbol{x}^{\prime}, \boldsymbol{y}^{\prime}\right\}$ of the orthogonal lattice $\left(L^{\prime}\right)^{\perp}$ to that lattice. Again, this is done by applying LLL to the lattice in $\mathbb{Z}^{\ell+2+\ell}$ generated by the rows of

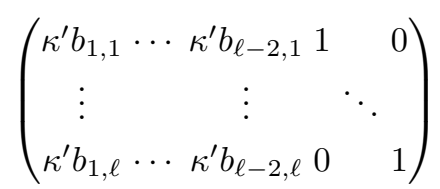

and keeping the last $\ell$ components of each resulting vector.

4. Enumerate the vectors $\boldsymbol{z}=a \boldsymbol{x}^{\prime}+b \boldsymbol{y}^{\prime} \in\left(L^{\prime}\right)^{\perp}$ of length at most $\sqrt{\ell N}$, and for each such vector $\boldsymbol{z}$, compute $\operatorname{gcd}(\boldsymbol{v}-\boldsymbol{z}, N)$ using all components, and return any nontrivial factor of $N$.

\subsection{Simulation Results}

Since the attack is heuristic, it is important to evaluate its experimental performances. To do so, we have implemented a simulation of the attack in SAGE 25]: for a given modulus $N$, we compute the vector $\boldsymbol{v}$ corresponding to a series of $\ell$ signatures on random messages and apply the lattice attack, attempting to recover a factor of $N$.

Table 1 shows the measured success probabilities for various values of $\ell$ and modulus sizes. It confirms the heuristic prediction that 5 faulty signatures should always suffice to factor $N$. It turns out that even 4 signatures are enough in almost half the cases.

Table 1. Attack success probability as a function of the number of faulty signatures and the size of $N$. Each parameter set was tested with random faults on 500 random moduli of the given size.

\begin{tabular}{|r|c|c|c|}
\hline Number of faulty signatures $\ell$ & 4 & 5 & 6 \\
\hline \hline 1024-bit moduli & $48 \%$ & $100 \%$ & $100 \%$ \\
\hline 1536-bit moduli & $45 \%$ & $100 \%$ & $100 \%$ \\
\hline 2048-bit moduli & $46 \%$ & $100 \%$ & $100 \%$ \\
\hline
\end{tabular}

Table 2. Efficiency of the attack with $\ell=5$ faulty signatures and various modulus sizes. Each parameter set was tested with random faults on 500 random moduli of the given size. Timings for a SAGE implementation, on a single $2.4 \mathrm{GHz}$ Core2 CPU core.

\begin{tabular}{|l|c|c|c|}
\hline Modulus size & 1024 & 1536 & 2048 \\
\hline \hline Average search space $\pi \ell N / V$ & 24 & 23 & 24 \\
\hline Average total CPU time & $16 \mathrm{~ms}$ & $26 \mathrm{~ms}$ & $34 \mathrm{~ms}$ \\
\hline
\end{tabular}


Experimental running times are given in Table 2. The whole attack takes a few dozen milliseconds on a standard PC. The number of vectors to test as part of the final exhaustive search step is about 20 in practice, which is done very quickly.

\section{Extending the Attack to Unknown Faulty Moduli}

As mentioned in 2.1 in its basic form, the attack requires the recovery of the faulty moduli $N_{i}^{\prime}$ in addition to the corresponding faulty signatures $\sigma_{i}^{\prime}$. This is not a very realistic assumption, since a typical implementation does not output the public modulus along with each signature.

To work around this limitation, we would like to reconstruct the vector $\boldsymbol{v}$ of integer values needed to run the attack from signatures alone, without the knowledge of the faulty moduli-possibly at the cost of requiring a few more faulty signatures.

This can actually be achieved in various ways depending on the precise form of the faults inflicted to the modulus. We propose solutions for the following two realistic fault models:

1. The faulty moduli $N_{i}^{\prime}$ differ from $N$ on a single (unknown) byte. This is known to be possible using power glitches or laser shots.

2. The differences between the faulty moduli $N_{i}^{\prime}$ and $N$ are located on the least significant half: the errors on the least significant bits can be up to half of the modulus size. It is easy to obtain such faults with a laser or a cold boot attack.

\subsection{Single Byte Faults}

In this model, the attacker is able to obtain a certain number $\ell^{\prime} \geq 5$ of pairs $\left(\sigma_{i}, \sigma_{i}^{\prime}\right)$ where $\sigma_{i}=\alpha x_{i}+\beta y_{i} \bmod N$ is a valid signature and $\sigma_{i}^{\prime}=\alpha x_{i}+\beta y_{i} \bmod$ $N_{i}^{\prime}$ is the same signature computed with a faulty modulus. The faulty moduli $N_{i}^{\prime}$ are not known, but they only differ from $N$ on a single byte whose position and value is unknown.

This type of fault can for example occur when attacking the transfer of the modulus to memory on a smart card with an 8-bit processor, or when using a laser attack with a sufficiently focused beam.

For a 1024-bit modulus $N$, for example, there are $128 \times 255 \approx 2^{15}$ possible faulty moduli. It can thus seem like a reasonable approach to try and run the attack with all possible faults. However, since this should be done with 5 signatures, this results in a search space of size $\approx\left(2^{15}\right)^{5}=2^{75}$ which is prohibitive.

This kind of exhaustive search can be made practical, though, if we take into account the fact that the CRT value $v_{i}=\mathrm{CRT}_{N, N_{i}^{\prime}}\left(\sigma_{i}, \sigma_{i}^{\prime}\right)$ satisfies:

$$
v_{i}=\alpha x_{i}+\beta y_{i} \leq N \cdot(p+q)=N^{3 / 2}\left(\sqrt{\frac{p}{q}}+\sqrt{\frac{q}{p}}\right)<(2 N)^{3 / 2}
$$


Table 3. Exhaustive search space size for the vector $\boldsymbol{v}$ of CRT values, and expected attack running time, depending on the number of pairs $\left(\sigma_{i}, \sigma_{i}^{\prime}\right)$ available to the attacker. Measured for a family of random single byte faults on a 1024-bit modulus. Timings are given for the SAGE implementation as above.

\begin{tabular}{|l|c|c|c|c|c|c|}
\hline Number of pairs $\ell^{\prime}$ & 5 & 7 & 10 & 15 & 20 & 25 \\
\hline \hline Search space size (bits) & 11.6 & 9.8 & 7.2 & 6.2 & 4.2 & 2.6 \\
\hline Total attack time (seconds) & 49 & 14 & 2.4 & 1.2 & 0.29 & 0.10 \\
\hline
\end{tabular}

since $p / q \in(1 / 2,2)$. Now, for a given value of $\sigma_{i}^{\prime}$, there are only very few possible target moduli $N_{i}^{*}$ differing from $N$ on a single byte such that $v_{i}^{*}=$ $\mathrm{CRT}_{N, N_{i}^{*}}\left(\sigma_{i}, \sigma_{i}^{\prime}\right)<(2 N)^{3 / 2}$ : often only one or two, and almost never more than 20 . We only need to run the attack with those target $v_{i}^{*}$ 's until we find a factor.

Experimentally, for a 1024-bit modulus, the average base 2 logarithm of the number of possible $v_{i}^{*}$ 's is about 2.5 , so if an attacker has 5 pairs $\left(\sigma_{i}, \sigma_{i}^{\prime}\right)$ in this model, they can expect to try all vectors $\boldsymbol{v}$ in a search space of around $12.5 \mathrm{bits}$, i.e. run the attack a few thousand times, for a total running time of under 2 minutes. This is already quite practical.

If more pairs are available, the attacker can keep the 5 pairs for which the number of possible $v_{i}^{*}$ 's is the smallest. This reduces the search space accordingly. In Table 3. we show how the exhaustive search space size and the expected running time evolve with the number of signatures in a typical example.

\subsection{Faults on Many Least Significant Bits}

In this model, the attacker is able to obtain $\ell=5$ signature families of the form $\left(\sigma_{i}, \sigma_{i, 1}^{\prime}, \ldots, \sigma_{i, k}^{\prime}\right)$, where the $\sigma_{i}$ 's are correct signatures:

$$
\sigma_{i}=\alpha x_{i}+\beta y_{i} \bmod N
$$

and the $\sigma_{i, j}^{\prime}$ 's are faulty signatures of the form:

$$
\sigma_{i, j}^{\prime}=\alpha x_{i}+\beta y_{i} \bmod N_{i, j}^{\prime} \quad 1 \leq i \leq \ell, 1 \leq j \leq k .
$$

In other words, for each one of the $\ell$ different messages, the attacker learns the reduction of the CRT value $v_{i}=\alpha x_{i}+\beta y_{i}$ modulo $N$, as well as modulo $k$ different unknown faulty moduli $N_{i, j}^{\prime}$. Additionally, it is assumed that all $N_{i, j}^{\prime}$ differ from $N$ only on the least significant bits, but the number of distinct bits can be as large as half of the modulus size: we assume that $\left|N-N_{i, j}^{\prime}\right|<N^{\delta}$ for a certain constant $\delta<1 / 2$.

This is a reasonable fault model for a laser attack: it suffices to target a laser beam on the least significant bits of $N$ to produce this type of faults.

To run the attack successfully, the attacker needs to recover the CRT values $v_{i}$. This can be done with high probability when the number of available faults $k$ for a given message is large enough. The simplest approach is based on a GCD computation. 
Indeed, fix an index $i \in\{1, \ldots, \ell\}$, and write $N_{i, j}^{\prime}=N+\varepsilon_{j}, v_{i}=u, \sigma_{i}=u_{0}$ and $\sigma_{i, j}^{\prime}=u_{j}$. The attacker knows the $u_{j}$ 's and wants to recover $u$.

Now, observe that there are integers $t_{j}$ such that $u$ satisfies $u=u_{0}+t_{0} \cdot N$ and $u=u_{j}+t_{j} \cdot\left(N+\varepsilon_{j}\right)$. In particular, for $j=1, \ldots, k$ we can write:

$$
\left(t_{j}-t_{0}\right) \cdot N+\left(u_{j}-u_{0}\right)+t_{j} \cdot \varepsilon_{j}=0 .
$$

This implies that $u_{j}-u_{0} \equiv t_{j} \cdot \varepsilon_{j}(\bmod N)$. However, we have $t_{j} \cdot \varepsilon_{j}<N^{1 / 2+\delta} \ll$ $N$, so that the congruence is really an equality in $\mathbb{Z}$. In view of (3), this implies that all $t_{j}$ 's are in fact equal, and hence:

$$
t_{0} \cdot \varepsilon_{j}=u_{0}-u_{j} \quad 1 \leq j \leq k .
$$

If the errors $\varepsilon_{j}$ on the modulus are co-prime, which we expect to happen with probability $\approx 1 / \zeta(k)$, we can then deduce $t_{0}$ as the GCD of all values $u_{0}-u_{j}$, and this gives:

$$
u=u_{0}+t_{0} \cdot N=u_{0}+N \cdot \operatorname{gcd}\left(u_{0}-u_{1}, \ldots, u_{0}-u_{k}\right) .
$$

As seen in Table 4, the success probability is in practice very close to $1 / \zeta(k)$ regardless of the size of errors.

It is probably possible to further improve the success probability by trying to remove small factors from the computed GCD $g=\operatorname{gcd}\left(u_{0}-u_{1}, \ldots, u_{0}-u_{k}\right)$ to find $t_{0}$ when $g>\sqrt{N}$, but we find that the number of required faults is already reasonable without this computational refinement.

Indeed, recall that $\ell=5 \mathrm{CRT}$ values are required to run the attack. If $k$ faults are obtained for each of the $\ell$ messages, the probability that these $\ell$ CRT values can be successfully recovered with this GCD approach is $\zeta(k)^{-\ell}$. This is greater than $95 \%$ for $k=7$, and $99 \%$ for $k=9$.

We can also mention an alternate, lattice-based approach to recovering the CRT value $u$. The relation between the different quantities above can be written in vector form as:

$$
u_{0} \mathbf{1}=\boldsymbol{u}+t_{0} \boldsymbol{e}
$$

Table 4. Success probabilities of the GCD method for CRT value recovery, depending on the number of available faults on a given message. Tested with random 1024-bit moduli. In the simulation, errors $\varepsilon_{j}$ are modeled as uniformly random signed integers of the given size, and 10,000 of them were generated for each parameter set.

\begin{tabular}{|r|c|c|c|c|}
\hline$k$ (faults per message) & 3 & 5 & 7 & 9 \\
\hline \hline $1 / \zeta(k)$ & .832 & .964 & .992 & .998 \\
\hline 100-bit errors & $83.2 \%$ & $96.8 \%$ & $99.0 \%$ & $99.8 \%$ \\
\hline 200-bit errors & $83.4 \%$ & $96.2 \%$ & $99.2 \%$ & $99.8 \%$ \\
\hline 400-bit errors & $82.7 \%$ & $96.6 \%$ & $99.1 \%$ & $99.8 \%$ \\
\hline Average CPU time & $.73 \mathrm{~ms}$ & $.75 \mathrm{~ms}$ & $.79 \mathrm{~ms}$ & $.85 \mathrm{~ms}$ \\
\hline
\end{tabular}


where $\mathbf{1}=(1, \ldots, 1), \boldsymbol{u}=\left(u_{1}, \ldots, u_{k}\right)$ and $\boldsymbol{e}=\left(\varepsilon_{1}, \ldots, \varepsilon_{k}\right)$.

Then, since $u_{0} \approx N$ is much larger than $\left\|t_{0} e\right\| \approx N^{1 / 2+\delta}$, short vectors orthogonal to $\boldsymbol{u}$ will be orthogonal to both $\mathbf{1}$ and $\boldsymbol{e}$. More precisely, we can heuristically expect that when $k$ is large enough $(k \gtrsim 2 /(1-2 \delta))$, the first $k-2$ vectors of a reduced basis of $\boldsymbol{u}^{\perp}$ will be orthogonal to $\mathbf{1}$ and $\boldsymbol{e}$.

Taking orthogonal lattices again, we can thus obtain a reduced basis $\{\boldsymbol{x}, \boldsymbol{y}\}$ of a two-dimensional lattice containing $\mathbf{1}$ and $\boldsymbol{e}$ (and of course $\boldsymbol{u}$ ). Since $\mathbf{1}$ is really short, we always find that $\boldsymbol{x}=\mathbf{1}$ in practice. Then, it happens quite often that $\boldsymbol{y}$ can be written as $\lambda \mathbf{1} \pm \boldsymbol{e}$, in which case $t_{0}$ is readily recovered as the absolute value of the second coordinate of $\boldsymbol{u}$ in the basis $\{\boldsymbol{x}, \boldsymbol{y}\}$.

However, this fails when $\mathbb{Z} \mathbf{1} \oplus \mathbb{Z} \boldsymbol{e}$ is a proper sublattice of $\mathbb{Z} \boldsymbol{x} \oplus \mathbb{Z} \boldsymbol{y}=\mathbb{Z}^{k} \cap$ $(\mathbb{Q} \mathbf{1} \oplus \mathbb{Q} \boldsymbol{e})$, namely, when there is some integer $d>1$ such that all errors $\varepsilon_{j}$ are congruent mod $d$. Thus, we expect the success probability of this alternate approach to be $1 / \zeta(k-1)$, which is slightly less than with the GCD approach.

\section{Practical Experiments}

Practical experiments for validating the new attack were done on an 8-bit $0.35 \mu \mathrm{m}$ RISC microcontroller with no countermeasures. As the microprocessor had no arithmetic coprocessor the values $\sigma_{p}$ and $\sigma_{q}$ were pre-computed by an external program upon each fault-injection experience and fed into the attacked device. The target combined $\sigma_{p}$ and $\sigma_{q}$ using multiplications and additions (using Formula 1) as well as the final modular reduction.

The location and spread of the faults were controlled by careful beam-size and shot-instant tuning. The reader is referred to the full version of this paper [9] for a description of the physical setting (common to the experiments reported in [16]).

We conducted several practical experiments corresponding to three different scenarios, roughly corresponding to the fault models considered in $\$ 2.1,33$ and $\$ 3.2$ respectively. Let us describe these experiments in order.

\subsection{First Scenario: Known Modulus}

In this case, we considered 5 messages for a random 1024-bit RSA modulus $N$. For each message $m_{i}$, we obtained a correct signature $\sigma_{i}$, as well as a faultymodulus signature $\sigma_{i}^{\prime}$ where the faulty modulus $N_{i}^{\prime}$ was also read back from the microcontroller.

Therefore, we were exactly in the setting described in $\$ 2.1$, and could apply the algorithm from $\$ 2.3$ directly: apply the Chinese Remainder Theorem to construct the vector $\boldsymbol{v}$ of CRT values and run the lattice-based attack to recover a factor of $N$.

The implementation of the attack used the same SAGE code as the simulation from 92.4 . In our experimental case, the ball of radius $\sqrt{N \ell}$ contained only about 10 vectors of the double orthogonal lattice, and the whole attack revealed a factor of $N$ in less than 20 milliseconds. 


\subsection{Second Scenario: Unknown Single Byte Fault}

In this case, we tried to replicate a setting similar to the one considered in \$3.1. We considered 20 messages and a random 1024-bit RSA modulus $N$. For each message $m_{i}$, we obtained a correct signature $\sigma_{i}$, as well as faulty-modulus signatures $\sigma_{i}^{\prime}$ with undisclosed faulty modulus $N_{i}^{\prime}$ generated by targeting a single byte of $N$ with the laser.

We had to eliminate some signatures, however, because in some cases, errors on the modulus turned out to exceed 8 bits 1 After discarding those, we had 12 pairs $\left(\sigma_{i}, \sigma_{i}^{\prime}\right)$ left to carry out the approach described in 3.1 .

The first step in this approach is to find, for each $i$, all values $v_{i}^{*}$ of the form $\mathrm{CRT}_{N, N_{i}^{*}}\left(\sigma_{i}, \sigma_{i}^{\prime}\right)\left(N_{i}^{*}\right.$ differing from $N$ only on one byte) that are small enough to be correct candidate CRT values. Unlike the setting of 3.1 , we could not assume that bit-differences were aligned on byte boundaries: we had to test a whole $1016 \times 255$ candidate modul $2 N_{i}^{*}$ for each $i$. Therefore, this search step was a bit costly, taking a total of 11 minutes and 13 seconds. Additionally, due to the higher number of candidate moduli, the number of candidate CRT values $v_{i}^{*}$ was also somewhat larger than in $\$ 3.1$, namely:

$$
7,17,3,9,15,5,14,44,44,17,10,55
$$

for our 12 pairs respectively. Keeping only the 5 indices with the smallest number of candidates, we obtained $3 \times 5 \times 7 \times 9 \times 10=9450$ possible $\mathrm{CRT}$ value vectors $v^{*}$.

We then ran the lattice-based attack on each of these vectors in order until a factor of $N$ was found. The factor was found at iteration number 2120, after a total computation time of 43 seconds.

\subsection{Third Scenario: Unknown Least Significant Bytes Faults}

In this case, we considered 10 messages for a random 1024-bit $N$. For each message $m_{i}$, we obtained a correct signature $\sigma_{i}$, as well as 10 faulty-modulus signature $\sigma_{i, j}^{\prime}$ with undisclosed faulty modulus $N_{i}^{\prime}$. The laser beam targeted the lower order bytes of $N$ but with a large aperture, generating multiple faults stretching over as much as 448 modulus bits.

In practice, we only used the data $\left(\sigma_{i}, \sigma_{i, 1}^{\prime}, \ldots, \sigma_{i, 10}^{\prime}\right)$ for the first 5 messages, discarding the rest. Then, we reconstructed the CRT values $v_{i}$ using the GCD technique of 3.2 .

\footnotetext{
${ }^{1}$ Note that in a real-world attack, it might not be possible to detect such overly spread out faults: hence, this particular technique should be used preferably when faults are known to affect only single bytes (e.g. in a glitch attack), whereas the technique from the next section is better suited to laser attacks as aperture control is much less of an issue.

${ }^{2}$ There are duplicates among those, corresponding to perturbations of 7 consecutive bits or less, but we did not attempt to avoid testing them several times, as this can only improve the search by a small constant factor while introducing significant complexity in the code.
} 


$$
v_{i}=\sigma_{i}+N \cdot \operatorname{gcd}\left(\sigma_{i}-\sigma_{i, 1}^{\prime}, \ldots, \sigma_{i}-\sigma_{i, 10}^{\prime}\right) \quad 1 \leq i \leq 5
$$

and applied the lattice-based attack on the resulting vector $\boldsymbol{v}$. This revealed a factor of $N$ in 16 milliseconds.

We also tried the same attack using a fewer number of the $\sigma_{i, j}^{\prime}$ 's, and found that it still worked when taking only 4 of those values in the computation of $v_{i}$ :

$$
v_{i}=\sigma_{i}+N \cdot \operatorname{gcd}\left(\sigma_{i}-\sigma_{i, 1}^{\prime}, \ldots, \sigma_{i}-\sigma_{i, 4}^{\prime}\right) \quad 1 \leq i \leq 5
$$

but failed if we took 3 instead. Considering that $1 / \zeta(3)^{5} \approx .40$ and $1 / \zeta(4)^{5} \approx .67$, this is quite in line with expectations.

\section{Countermeasures and Further Research}

Probabilistic and stateful signature schemes are usually secure against this attack, since they make it difficult to obtain two signatures on the same padded message. However, all deterministic schemes are typically vulnerable, including those in which the attacker doesn't have full access to the signed message, provided that the target device can be forced to compute the same signature twice.

A natural countermeasure is to use a CRT interpolation formula that does not require $N$, such as Garner's formula, computed as follows:

$$
\begin{array}{|l|}
\hline t \leftarrow \sigma_{p}-\sigma_{q} \\
\text { if } t<0 \text { then } t \leftarrow t+p \\
\sigma \leftarrow \sigma_{q}+(t \cdot \gamma \bmod p) \cdot q \\
\operatorname{return}(\sigma)
\end{array}
$$

where we assume that $p>q$, and $\gamma$ is the usual CRT coefficient $q^{-1} \bmod p$. Note that the evaluation of $\sigma$ does not require a modular reduction because

$$
\sigma=\sigma_{q}+(t \cdot \gamma \bmod p) \cdot q \leq q-1+(p-1) q<N
$$

Besides the obvious countermeasures consisting in checking signatures before release, it would be interesting to devise specific countermeasures for protecting Formula (11) (or Garner's formula) taking into account the possible corruption of all data involved.

Finally, in a number of special cases and particular settings (e.g. Appendix A) other fault attacks on the CRT recombination phase can be devised. A thorough analysis of such scenarios is also an interesting research direction.

Acknowledgments. We would like to thank the anonymous referees for helpful comments. The work described in this paper has been supported in part by the European Commission through the ICT program under contract ICT-2007216676 ECRYPT II. 


\section{References}

1. Aumüller, C., Bier, P., Fischer, W., Hofreiter, P., Seifert, J.-P.: Fault Attacks on RSA with CRT: Concrete Results and Practical Countermeasures. In: Kaliski Jr., B.S., Koç, Ç.K., Paar, C. (eds.) CHES 2002. LNCS, vol. 2523, pp. 260-275. Springer, Heidelberg (2003)

2. Bellare, M., Rogaway, P.: The exact security of digital signatures - how to sign with RSA and rabin. In: Maurer, U.M. (ed.) EUROCRYPT 1996. LNCS, vol. 1070, pp. 399-416. Springer, Heidelberg (1996)

3. Berzati, A., Canovas, C., Dumas, J.-G., Goubin, L.: Fault attacks on RSA public keys: Left-to-right implementations are also vulnerable. In: Fischlin, M. (ed.) CT-RSA 2009. LNCS, vol. 5473, pp. 414-428. Springer, Heidelberg (2009)

4. Berzati, A., Canovas, C., Goubin, L.: Public key perturbation of randomized RSA implementations. In: Mangard, S., Standaert, F.-X. (eds.) CHES 2010. LNCS, vol. 6225, pp. 306-319. Springer, Heidelberg (2010)

5. Berzati, A., Canovas, C., Goubin, L.: Perturbating RSA public keys: An improved attack. In: Oswald, E., Rohatgi, P. (eds.) CHES 2008. LNCS, vol. 5154, pp. 380395. Springer, Heidelberg (2008)

6. Boneh, D., DeMillo, R.A., Lipton, R.J.: On the importance of eliminating errors in cryptographic computations. J. Cryptology 14(2), 101-119 (2001)

7. Boscher, A., Naciri, R., Prouff, E.: CRT RSA algorithm protected against fault attacks. In: Sauveron, D., Markantonakis, K., Bilas, A., Quisquater, J.-J. (eds.) WISTP 2007. LNCS, vol. 4462, pp. 229-243. Springer, Heidelberg (2007)

8. Brier, E., Chevallier-Mames, B., Ciet, M., Clavier, C.: Why one should also secure RSA public key elements. In: Goubin, L., Matsui, M. (eds.) CHES 2006. LNCS, vol. 4249, pp. 324-338. Springer, Heidelberg (2006)

9. Brier, E., Naccache, D., Nguyen, P.Q., Tibouchi, M.: Modulus Fault Attacks Against RSA-CRT Signatures. Full version of this paper. Cryptology ePrint Archive, http://eprint.iacr.org/

10. Ciet, M., Joye, M.: Practical fault countermeasures for Chinese remaindering based cryptosystems. In: Breveglieri, L., Koren, I. (eds.) FDTC, pp. 124-131 (2005)

11. Coppersmith, D.: Small solutions to polynomial equations, and low exponent RSA vulnerabilities. J. Cryptology 10(4), 233-260 (1997)

12. Coron, J.-S., Joux, A., Kizhvatov, I., Naccache, D., Paillier, P.: Fault attacks on RSA signatures with partially unknown messages. In: Clavier, C., Gaj, K. (eds.) CHES 2009. LNCS, vol. 5747, pp. 444-456. Springer, Heidelberg (2009)

13. Coron, J.-S.: Optimal security proofs for PSS and other signature schemes. In: Knudsen, L.R. (ed.) EUROCRYPT 2002. LNCS, vol. 2332, pp. 272-287. Springer, Heidelberg (2002)

14. Giraud, C.: An RSA implementation resistant to fault attacks and to simple power analysis. IEEE Trans. Computers 55(9), 1116-1120 (2006)

15. Lenstra, A.K., Lenstra Jr., H.W., Lovász, L.: Factoring polynomials with rational coefficients. Math. Ann. 261(4), 515-534 (1982)

16. Mirbaha, A.-P., Dutertre, J.M., Tria, A., Agoyan, M., Ribotta, A.-L., Naccache, D.: Study of single-bit fault injection techniques by laser on an AES cryptosystem. In: Gizopoulos, D., Chatterjee, A. (eds.) IOLTS (2010)

17. Muir, J.A.: Seifert's RSA fault attack: Simplified analysis and generalizations. In: Ning, P., Qing, S., Li, N. (eds.) ICICS 2006. LNCS, vol. 4307, pp. 420-434. Springer, Heidelberg (2006) 
18. Nguyen, P.Q.: Public-key cryptanalysis. In: Luengo, I. (ed.) Recent Trends in Cryptography. Contemporary Mathematics, vol. 477. AMS-RSME (2009)

19. Nguyên, P.Q., Stern, J.: Merkle-Hellman Revisited: A Cryptanalysis of the QuVanstone Cryptosystem Based on Group Factorizations. In: Kaliski Jr., B.S. (ed.) CRYPTO 1997. LNCS, vol. 1294, pp. 198-212. Springer, Heidelberg (1997)

20. Nguyên, P.Q., Stern, J.: Cryptanalysis of a fast public key cryptosystem presented at SAC 1997. In: Tavares, S., Meijer, H. (eds.) SAC 1998. LNCS, vol. 1556, pp. 213-218. Springer, Heidelberg (1999)

21. Nguyên, P.Q., Stern, J.: The two faces of lattices in cryptology. In: Silverman, J.H. (ed.) CaLC 2001. LNCS, vol. 2146, pp. 146-180. Springer, Heidelberg (2001)

22. Rivain, M.: Securing RSA against fault analysis by double addition chain exponentiation. In: Fischlin, M. (ed.) CT-RSA 2009. LNCS, vol. 5473, pp. 459-480. Springer, Heidelberg (2009)

23. Rivest, R.L., Shamir, A., Adleman, L.M.: A method for obtaining digital signatures and public-key cryptosystems. Commun. ACM 21(2), 120-126 (1978)

24. Seifert, J.-P.: On authenticated computing and rsa-based authentication. In: Atluri, V., Meadows, C., Juels, A. (eds.) ACM Conference on Computer and Communications Security, pp. 122-127. ACM, New York (2005)

25. Stein, W.A., et al.: Sage Mathematics Software (Version 4.4.2). The Sage Development Team (2010), http://www.sagemath.org

26. Vigilant, D.: RSA with CRT: A new cost-effective solution to thwart fault attacks. In: Oswald, E., Rohatgi, P. (eds.) CHES 2008. LNCS, vol. 5154, pp. 130-145. Springer, Heidelberg (2008)

\section{A Using Dichotomy in the Absence of Padding}

Consider again the setting of \$2.1, in which an adversary is able to obtain both a correct signature $\sigma$ on a message $m$, and a signature on the same message $m$ computed with a faulty modulus, allowing him to deduce the non reduced value $v=\sigma_{p} \cdot \alpha+\sigma_{q} \cdot \beta \in \mathbb{Z}$. We can write:

$$
v=(\sigma \bmod p) \cdot \alpha+(\sigma \bmod q) \cdot \beta=\left(\sigma-p\left\lfloor\frac{\sigma}{p}\right\rfloor\right) \cdot \alpha+\left(\sigma-q\left\lfloor\frac{\sigma}{q}\right\rfloor\right) \cdot \beta
$$

Moreover, observe that $\alpha+\beta=N+1$ (as is easily seen by reducing $\alpha+\beta$ modulo $p$ and $q$ ). Therefore, we have:

$$
v=\sigma \cdot(N+1)-p \alpha\left\lfloor\frac{\sigma}{p}\right\rfloor-q \beta\left\lfloor\frac{\sigma}{q}\right\rfloor
$$

Hence, if we let $\omega=(\sigma \cdot(N+1)-v) / N$, we get:

$$
\omega=\frac{\sigma \cdot(N+1)-v}{N}=\frac{\alpha}{q}\left\lfloor\frac{\sigma}{p}\right\rfloor+\frac{\beta}{p}\left\lfloor\frac{\sigma}{q}\right\rfloor
$$

and this value $\omega$ is an integer since $v \equiv \sigma(\bmod N)$. 
Now assume further that the adversary can ask signatures on messages $m$ such that $\sigma$ is small. This is the case, for example, when signatures are computed without padding and the physical device under consideration will answer arbitrary signature queries: then, the adversary can simply ask signatures on messages of the form $\sigma^{e}$ for small values $\sigma$ of his choice.

In such a setting, the adversary can pick a $\sigma$ close to $N^{1 / 2}$, carry out the fault attack and compute the integer $\omega$. By (4), he gets $\omega=0$ if $\sigma<\min (p, q)$ and $\omega>0$ otherwise. Trying this process again several times, the smallest prime factor of $N$ can be recovered by dichotomy. 